\title{
SIMPLE AND ROBUST EYE MOVEMENTS DETECTION METHOD
}

\author{
FARS ESMAT SAMANN \\ Dept. of Electrical and Computer Engineering, University of Duhok, Kurdistan Region-Iraq
}

\begin{abstract}
The aim of this research is to develop a signal processing method to detect four eye movements, such as looking up, down, left, right and blinking. This new method has a couple of features in comparison with the recent eye movement detection algorithms. Most of the recent algorithms require a De-noising stage, which is not required in this work. In addition, the suggested algorithm can be considered simple and robust in noisy environment in contrast with other algorithms. In this paper, short-time averaging method is proposed to process and to extract parameters from EOG signals. Moreover, adaptive threshold is applied to classify EOG pulses. The purpose of the adaptive threshold is to enhance the performance of the algorithm in a noisy background. Simulation results are based on real-life EOG signals, where these signals were recorded using an Electrooculography system. The results show that the proposed algorithm has a stable performance $\mathrm{HR}=100 \%$ and $\mathrm{FR}=0 \%$ with $\mathrm{SNR}$ greater than $2 \mathrm{~dB}$. The average performance with $\mathrm{SNR}=0.5 \mathrm{~dB}$ is about $\mathrm{HR}=\mathbf{9 0 . 2 1 \%}$ and $\mathrm{FR}=\mathbf{4 . 8 8 \%}$.
\end{abstract}

KEYWORDS: Electrooculogram (EOG), Short-Time Averaging (STA)

\section{INTRODUCTION}

$\mathbf{B}_{\text {is }}^{\text {is }}$ io signal like EOG has attracted the attention of many researchers recently because it has potential to help disabled people in controlling wheel chair and in using their computer (Estrany, Fuster, Garcia, \& Luo , 2009). In order to measure Bio signals generated by eye movements, Electrooculography has been used to measure the bio-potential generated by the changes in the positioning of the eye. The measured signals are called the Electrooculogram (EOG). Usually, the EOG signals before amplification have relatively small voltage between 15 to 200 microvolts (Malik \& Ahmad, 2007), so they are very sensitive to interference. In addition, during a long EOG recording session, the recorded EOG signals are usually distorted by noise caused by the high impedance existed between the skin and electrode due to the sweat and skin oil. Therefore, it is difficult to identify EOG signals without De-noising stage that minimizes the interference (Jiang \& Zhou, 2013). Several of De-noise methods have been suggested to enhance the accuracy in recognizing eye movements. Mathematical morphology filtering method has been recommended to process the EOG signals in time domain to achieve average recognition rate of 93\% (Jiang \& Zhou, 2013). Moreover, stationary wavelet transform with hard/soft thresholding method is proposed to De- noise unprocessed EOG signals (Rajesh A, S., T., \& K., 2012). This technique is based on transforming time domain signal to frequency domain. Furthermore, the effectiveness of this method depends on the type of wavelet and decomposition wavelet (Mala \& Latha, 2016). Most of the recent algorithms of eye movement detection are based on differential method (Merino, Rivera, Gómez, Molina, \& Dorronzoro, 2010) (Jiang \& Zhou, 2013). In other words, the derivative of the EOG signal is considered to detect eye movements. The problem with these algorithms is that they require a perfect De-noise stage because noise has significant impact on the accuracy of these algorithms. This paper describes a simple method for detecting the direction of eye movements without any De-noising stage. This paper is organized in the following manner: EOG signals, Data Acquisition, EOG signal processing, thresholding and classification process, Experiment and Results, and Conclusion

\section{EOG SIGNALS}

Usually, the cornea of the eye has voltage of +0.4 to +1.0 millivolts which is considered higher than the retina (Jose, 2013). This potential difference between the retina and cornea is due to the presence of active nerves in the retina compared to the front of the eye (Estrany, Fuster, Garcia, \& Luo , 2008). Moreover, the potential 
can be considered as an electrical dipole signal (Kim \& Yoon, 2013). This signal has a negative pole at retina and a positive pole at the cornea as shown

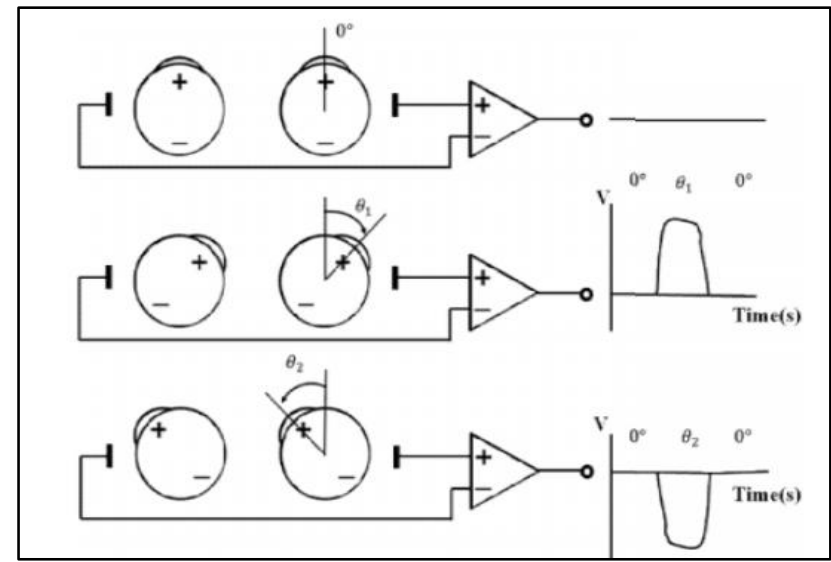

Fig. (1): Output of EOG signal for looking straight (TOP), looking right direction (MIDDLE), looking left direction (BOTTOM) (Jose, 2013)

When the eyeball is rolling upward or in the right direction, positive pulses are generated in the vertical and horizontal channels. When the eyeball is rolling downward or towards the left direction, negative pulses are generated in vertical and horizontal channels. During blinking, positive pulses with small duration and high amplitudes are generated in the vertical channel only. The duration of EOG pulses is proportional to the process of eyeball rolling. Typically EOG signals have frequency band from 0.05 to $50 \mathrm{~Hz}$ (Iáñez, Úbeda, \& Azorín, 2011). The average duration of different eye movements is located in table 1 (Jose, 2013).

Table 1: Duration of eye movements in millisecond (Jose, 2013)

\begin{tabular}{cccc}
\hline Eyeball movements & Polarity & Pulse duration & Channel \\
\hline Upward & positive & $400-600 \mathrm{~ms}$ & Vertical Channel \\
\hline Downward & negative & $400-600 \mathrm{~ms}$ & Vertical Channel \\
\hline Blinking & positive & $250-300 \mathrm{~ms}$ & Vertical Channel \\
\hline Right & positive & $400-600 \mathrm{~ms}$ & Horizontal Channel \\
\hline Left & negative & $400-600 \mathrm{~ms}$ & Horizontal Channel \\
\hline
\end{tabular}

The following block diagram shows the stages of the suggested eye movement detection algorithm. The acquired EOG signals from the vertical and horizontal channels are processed with Short-Time averaging method and then it is fed up to thresholding and classification stages. 


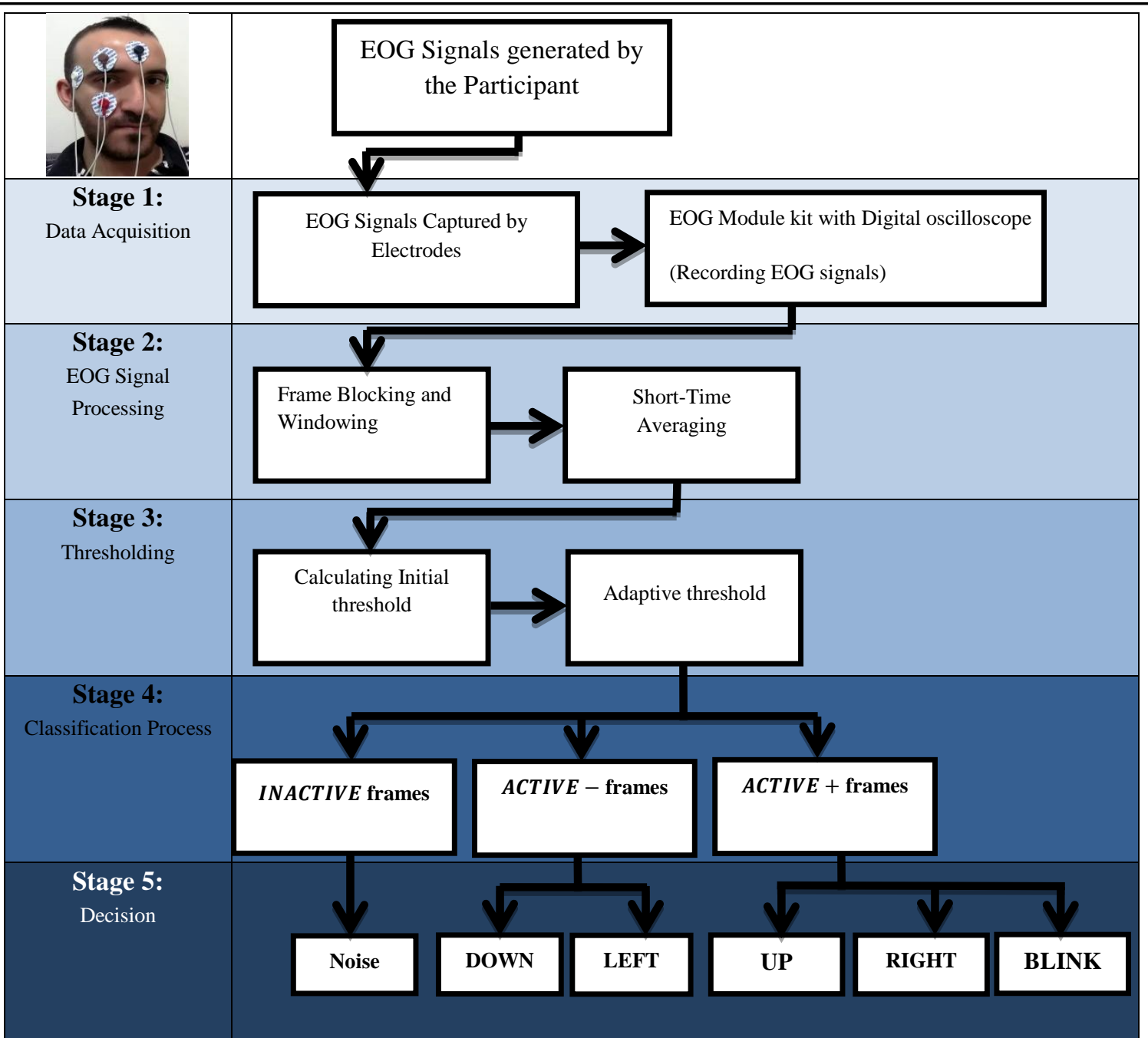

Fig. (2): Block Diagram of eye movement detection algorithm

\section{DATA ACQUISITION}

A system of four disposable $\mathrm{Ag} / \mathrm{AgCl}$ electrodes is placed on the participant to measure the potential difference of eye movements in the vertical and horizontal channels (see Fig. 3). These electrodes were used to capture the EOG signals from the participant.

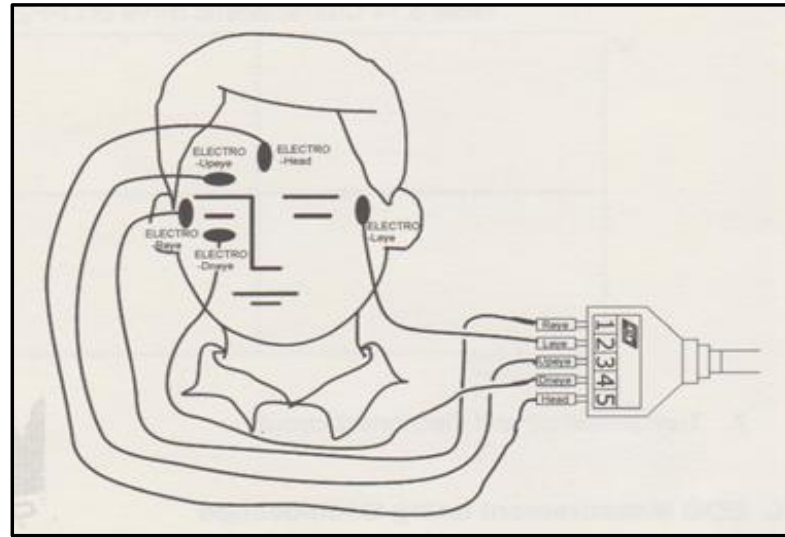

Fig. (3): Locations of $\mathrm{Ag} / \mathrm{AgCl}$ electrodes around eyes 
The EOG signals are measured using KL75003 Electrooculogram Module. This Module consists of four stages for each vertical and horizontal channel as shown in Figure 4.

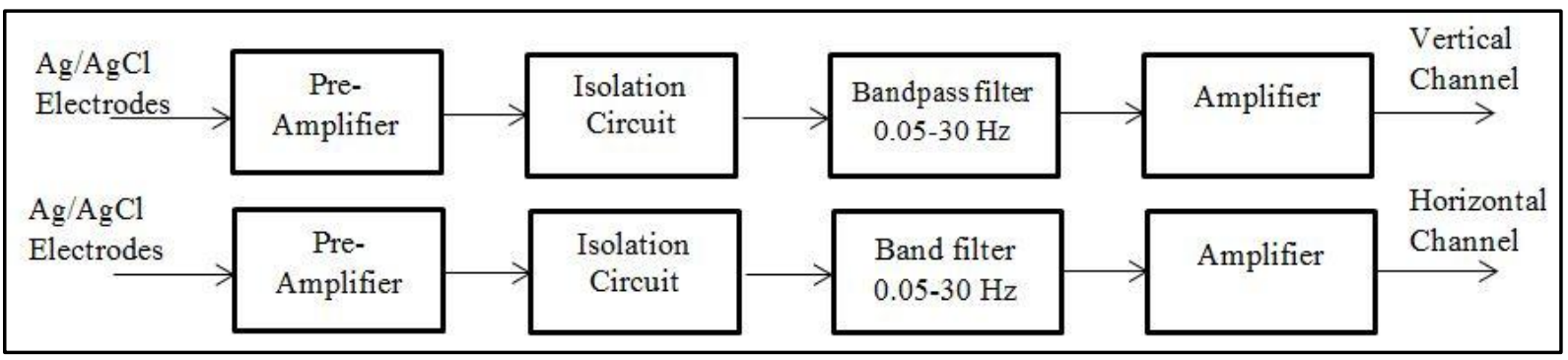

Fig. (4): Block diagram of KL-75003 EOG Module

This EOG Module has a bandpass filter ( 0.05 $\mathrm{Hz}$ to $30 \mathrm{~Hz}$ ) with two stages of amplification to amplify EOG signal. The range of amplitude that can be obtained from KL-75003 EOG Module is between $500 \mathrm{mV}$ to $1.1 \mathrm{~V}$. The Tektronix oscilloscope is used to record the EOG signals with a sampling rate of 500 samples/sec in the DC coupling mode. The recorded signals are saved as an excel sheet, and this excel sheet is imported into MATLAB. In this paper, MATLAB has been used to simulate the proposed algorithm.

\section{EOG SIGNAL PROCESSING}

\subsection{Frame Blocking and Windowing}

In order to ensure the stationary of noise ( $\mathrm{Lv}$, Wu, Li, \& Zhang, 2010), frame blocking method is used, where the EOG signal is block into frames of $\mathrm{N}$ samples and overlapped with $\mathrm{M}$ samples of the adjacent frames as illustrated in figure 5. The length of the frames has been chosen regarding to the properties of the EOG signals. In EOG signals, the duration of blink pulses is between 250-300 msec and the duration of eye movements (for example looking up or down) is 400-600 msec. Therefore, the duration of frame is chosen to be $100 \mathrm{msec}$ with overlapping $20 \mathrm{msec}$ to extract most of the features of EOG signals. The number of samples per frame is calculated as follows:

$N=$

Fs ( samples $/ \mathrm{sec}) \times$ duration of Frame in sec (1)

$N=500$ samples $/ \mathrm{sec} \times 0.1 \mathrm{sec}$

$N=50$ samples per frame

In the same way, $\mathrm{M}$ is calculated ( $\mathrm{M}=10$ samples).

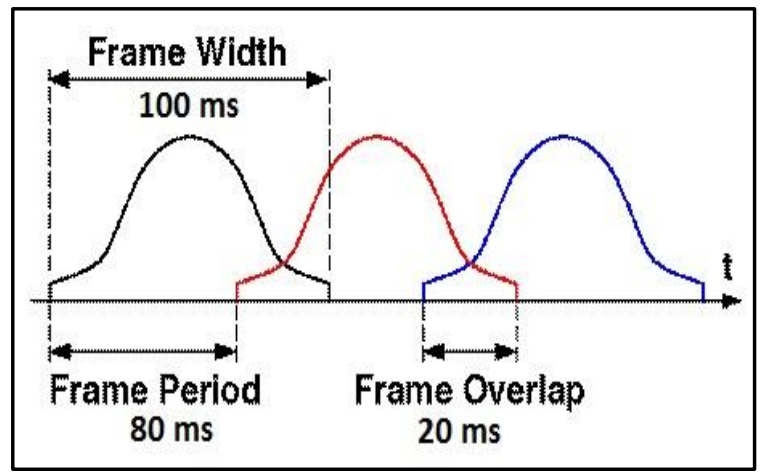

Fig. (5): Data windowing and frame formation

After blocking the EOG signals into frames, hamming window is applied to each individual frame to reduce the discontinuity at the beginning and at the end of each frame (see Fig. 5). In this case, $x(n)$ refers to finite duration fragment of EOG signal.

$\check{x}(n)=x(n) \times w(n)$ 
Where $\check{x}(n)$ is the windowed signal and $w(n)$ is used to symbolize hamming window. It is defined as the following:

$$
w(n)= \begin{cases}0.54-0.46 \cos \left[\frac{2 \pi n}{N-1}\right], & 0 \ll n \ll N-1 \\ 0, & \text { otherwise }\end{cases}
$$

\subsection{Short-Time Averaging method}

The average of a frame is the most important parameter of the proposed algorithm. The ShortTime Averaging (STA) method is similar to the Short-Time Energy method, which is commonly used for Voice Active Detection algorithms (Sangwan, M.C, Jamadagni, Sah, Prasad, \& Gaurav, 2002). The proposed method is basically working as a moving average filter by calculating the average value of individual frames to give more representative intermediate waveform value. The main reason for using a moving average filter is to smooth out the EOG waveform from the low and high aberrations. The smoothing factor can be controlled by the number of samples per frame (N). A higher smoothing factor gives better smoothing constraints comparing with lower values (see figure 6). However, the properties of the EOG pulses should be taken in consideration in choosing the length of frame $\mathrm{N}$ (see subsection 4.1) because choosing very high smoothing factor may lead to lose most of the features of EOG signals (see figure 6-D).

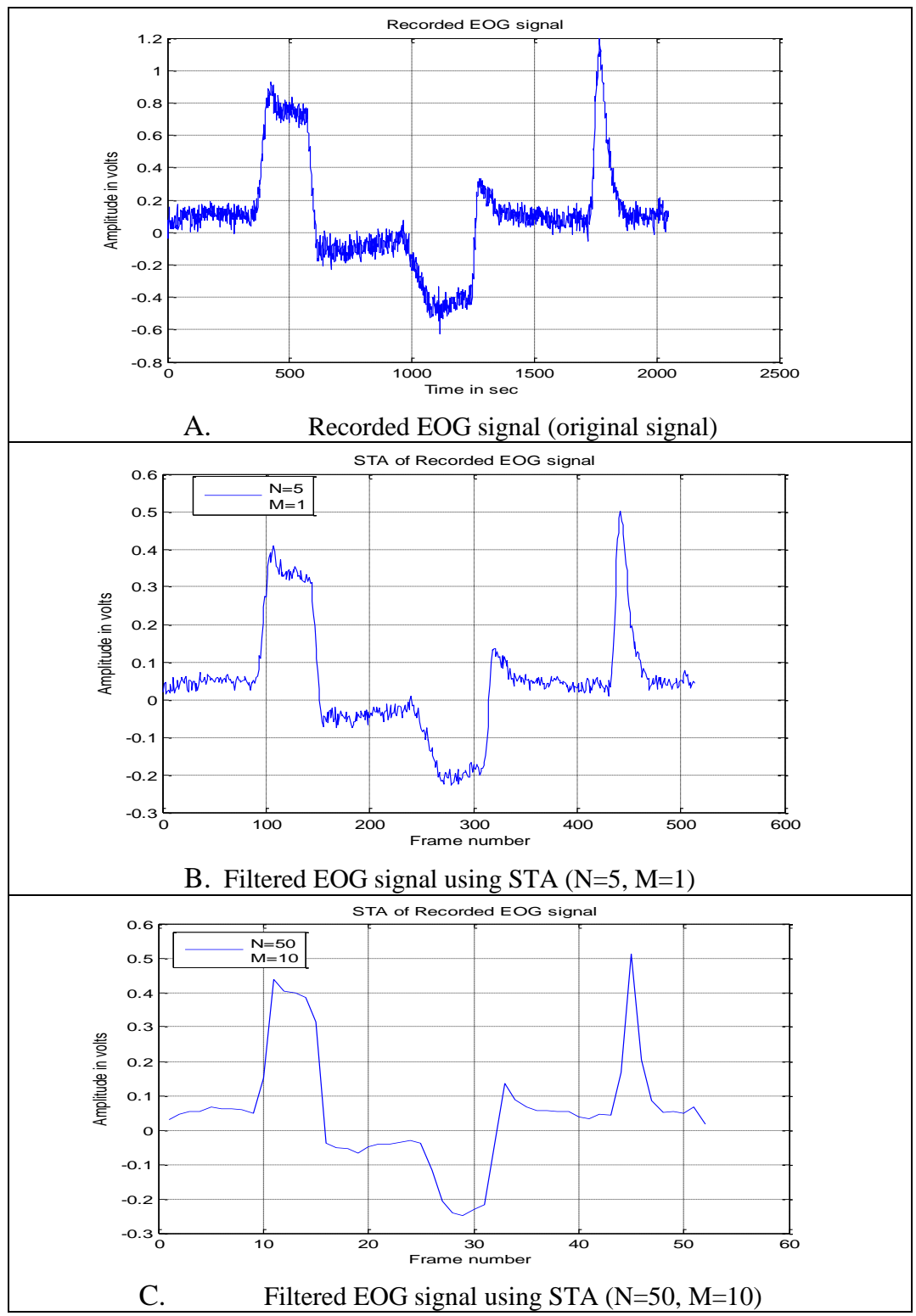




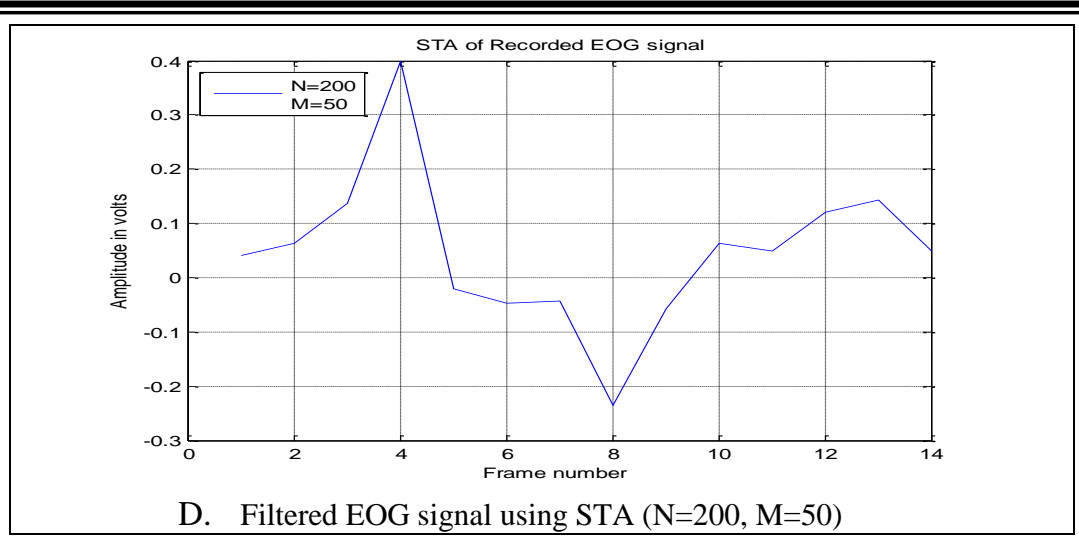

Fig. (6): EOG signal filtered by STA using high and low smoothing factor.

Let $x(n)$ be the $n^{\text {th }}$ frame of EOG signal. If the length of the frame was $\mathrm{N}$ samples with $\mathrm{M}$ samples overlapped, then the average of a frame can be represented in time domain as follows:

$A V_{n}=\frac{1}{N} \sum_{i=(n-1)(N-m)}^{n N-m(n-1)} x(i)$

Where $A V_{n}$ is the average of $\mathrm{n}^{\text {th }}$ frame.

\section{THRESHOLDING AND CLASSIFICATION PRCOESS}

\subsection{Initial value of threshold}

To track the background noise, the initial value of the threshold should be obtained to evaluate the beginning of the threshold ( Prasad, Sangwan, Jamadagni, M.C, \& Sah, 2002). The absolute values of the first five frames (the first $500 \mathrm{msec}$ is assumed to be noise) are stored in a buffer which maintains noise frames only. The initial threshold is calculated by taking the average of the buffer $\mathrm{b}(\mathrm{i})$ as follows:

$$
A V_{t h}=\frac{1}{v} \sum_{i=1}^{v} b(i)
$$

Where, $A \mathrm{~V}_{\text {th }}=$ initial threshold estimate

$$
\mathrm{v}=\text { number of frames stored in the buffer }
$$

The variance of the initial threshold is obtained by $\sigma=\operatorname{VAR}\{\mathrm{b}(\mathrm{i})\}$

\subsection{Classification process}

The average of the frame value is considered for classification process. Frames may be classified as 'INACTIVE' (noise) or 'ACTIVE' (EOG signal). The ACTIVE frames are sub classified into 'ACTIVE+' and 'ACTIVE-' regarding to the polarity of the frame. This classification is based on that the value of ACTIVE+ frames is greater than the INACTIVE frames, and the value of ACTIVE- frames is less than the INACTIVE frames. The classification method has been used for Voice Active Detection algorithm, and it can be found in (Gold \& Morgan,
1999). In this paper, the rule of classification is updated as follows:

IF $\left(A V_{n}>k A V_{t h}\right)$

where $k>1$

Frame is ACTIVE +

ELSE IF $\left(A V_{n}<-k A V_{t h}\right)$

Frame is ACTIVE -

ELSE

Frame is INACTIVE

Regarding the aforementioned classification, $\mathbf{A V}_{\text {th }}$ is considered to be the average of noise frames, while $\boldsymbol{k} \boldsymbol{A} \boldsymbol{V}_{\boldsymbol{t h}}$ is used as a threshold to make decisions. Where ' $\boldsymbol{k}$ ' is a scaling factor, which allows safe band of adaptation. However, a fixed threshold can be considered 'deaf' to the variation of background noise. Therefore, adaptive threshold will be more effective.

\subsection{Adaptive linear Average-based thresholding}

The following adaptive thresholding is suggested by ( Prasad, Sangwan, Jamadagni, M.C, \& Sah, 2002). Since the background noise is nonstationary, an adaptive thresholding method is more appropriate. Regarding the classification, each time an INACTIVE frame is detected the buffer will be updated. The new INACTIVE frame is added to the buffer, and the oldest is removed. After updating the buffer, the variance and the mean of the buffer are re-calculated, and they are denoted as $\boldsymbol{\sigma}_{\text {new }}$ and $\mathbf{A} \mathbf{V}_{\text {new }}$.Finally, the threshold value will be updated by using Eq.(7); the formula to update the threshold is given by (Pollak, Sovka, \& Jan, 1993):

$A V_{\text {th-new }}=(1-p) k A V_{\text {th-old }}+p k A V_{\text {new }}$

Where, $\boldsymbol{k} \boldsymbol{A} \boldsymbol{V}_{\boldsymbol{t h - o l d}}$ is the previous threshold value $\boldsymbol{A} \boldsymbol{V}_{\text {new }}$ is the average of most recent noise frames $\boldsymbol{A} \boldsymbol{V}_{\text {th-new }}$ is the updated threshold value

In case of a sudden change in the background noise, the new variance $\boldsymbol{\sigma}_{\text {new }}$ will be greater than the previous one $\boldsymbol{\sigma}_{\mathbf{o l}}$. This will result in an update of the value 
Table (2): Variation of $p$ regarding to the ratio of $\sigma_{\text {new }}$ and $\sigma_{\text {old }}$ ( Prasad, Sangwan, Jamadagni, M.C, \& Sah, 2002)

\begin{tabular}{|c|c|}
\hline$\frac{\sigma_{\text {new }}}{\sigma_{\text {old }}} \geq 1.25$ & $\mathrm{p}=0.25$ \\
\hline $1.25 \geq \frac{\sigma_{\text {new }}}{\sigma_{\text {old }}} \geq 1.10$ & $\mathrm{p}=0.20$ \\
\hline $1.10 \geq \frac{\sigma_{\text {new }}}{\sigma_{\text {old }}} \geq 1.0$ & $\mathrm{p}=0.15$ \\
\hline $1.00 \geq \frac{\sigma_{\text {new }}}{\sigma_{\text {old }}}$ & $\mathrm{p}=0.10$ \\
\hline
\end{tabular}

The decision by the proposed algorithm for identifying four eye movements is based on the number of $\boldsymbol{A C T I V E}+$ and $\boldsymbol{A C T I V E}$ - frames detected for each EOG pulse in the vertical and horizontal channels as illustrated in the following flow chart.
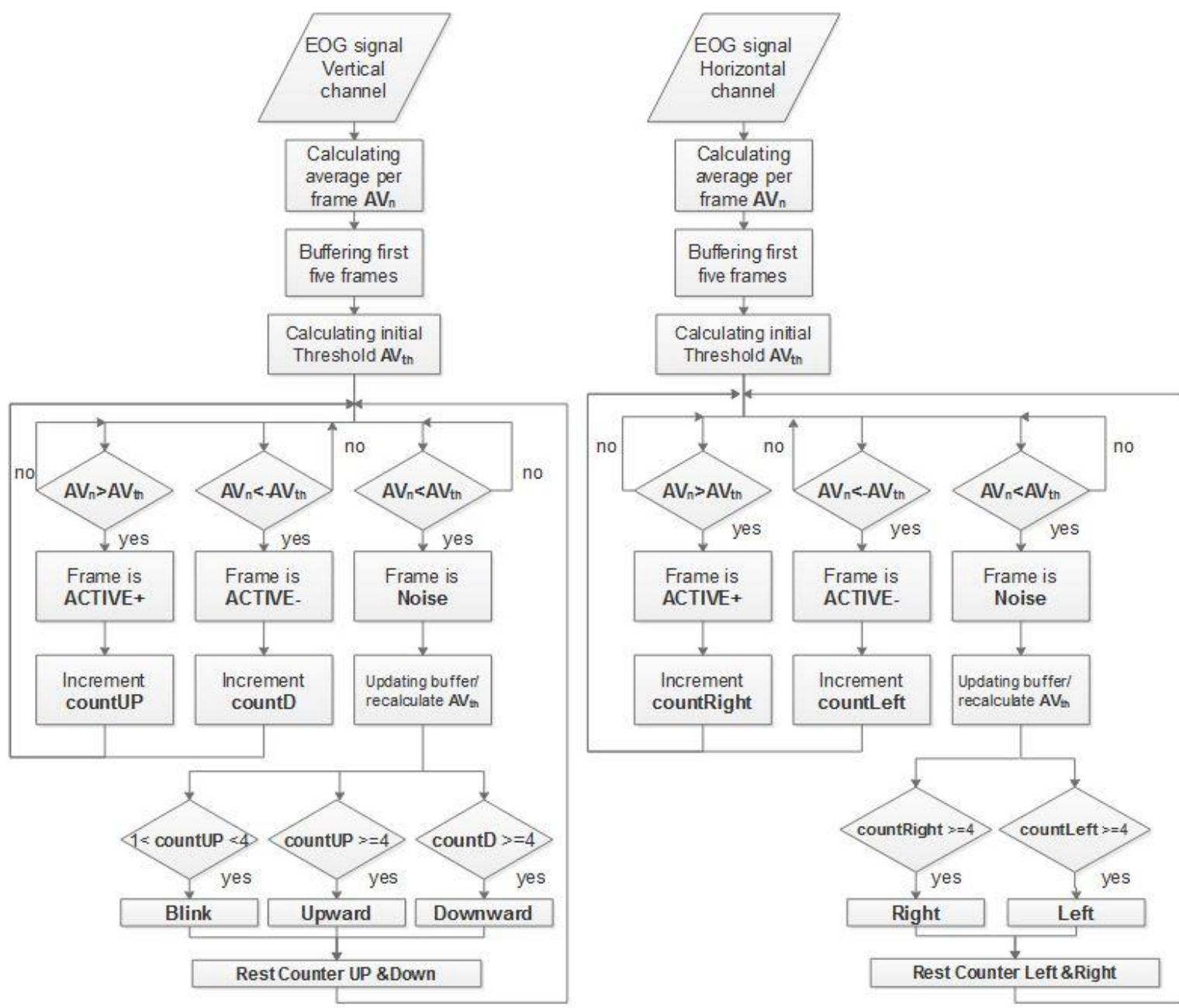

Fig. (7): Flow chart of the proposed eye movement detection algorithm

\section{EXPERIMENT AND RESULTS}

Six participants participated in testing this algorithm. Each of the participants were asked to move their eyes to extreme side Up/Right/Down/Left and returned to the initial position for three times in a row respectively. Additionally, they were asked to blink for three times in a row. Sequences of three EOG pulses representing blinking, Right, Left, Up and Down movements respectively were recorded from each of the participants individually (see figure 8). 


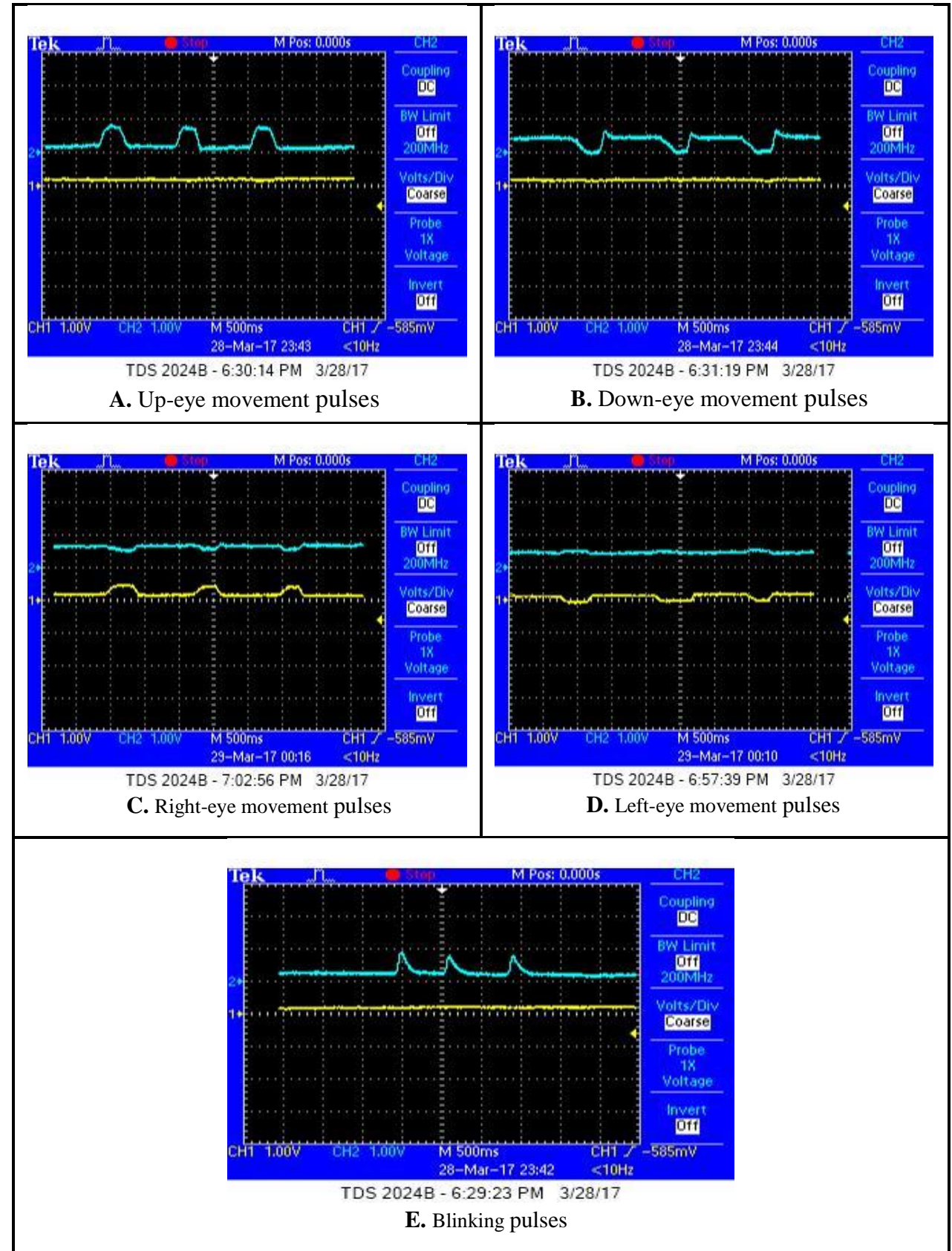

Fig. (8): Recorded EOG pulses (Blue line represents Vertical channel, and Yellow line represents Horizontal channel)

The purpose of recording a sequence of three pulses from each participant for each eye movement is to obtain different amplitudes and durations of the EOG pulses in order to test the reliability of this algorithm. With Regards to the results, the number of frames per EOG pulse is located in Table 3.

Table (3): Duration of eye movements in terms of frames

\begin{tabular}{cc}
\hline Eyeball movements & Duration range (No. of frames) \\
\hline Blinking & 2 to 3 \\
\hline Looking Up & 4 to 6 \\
\hline Looking Down & 4 to 6 \\
\hline Looking Right & 4 to 6 \\
\hline Looking Left & 4 to 6 \\
\hline
\end{tabular}


It is found that the duration of blinking pulses consist of two or three frames per pulse, and the duration of Looking up, down, right and left is between four to six frames per pulse. These values were considered in this algorithm to distinguish between looking upward pulses and blinking pulses in the vertical channel. Moreover, in order to test the robustness of the suggested algorithm in a noisy background, a white Gaussian noise has been added to the recorded EOG signals. The following SNRs were considered $15 \mathrm{~dB}, 10 \mathrm{~dB}$, $5 \mathrm{~dB}, 2 \mathrm{~dB}, 1 \mathrm{~dB}$ and $0.5 \mathrm{~dB}$. Figure $9 \& 10$ shows the difference between the recorded EOG signal and the processed EOG signal with STA method in case of $15 \mathrm{~dB}$ and $0.5 \mathrm{~dB}$ respectively.
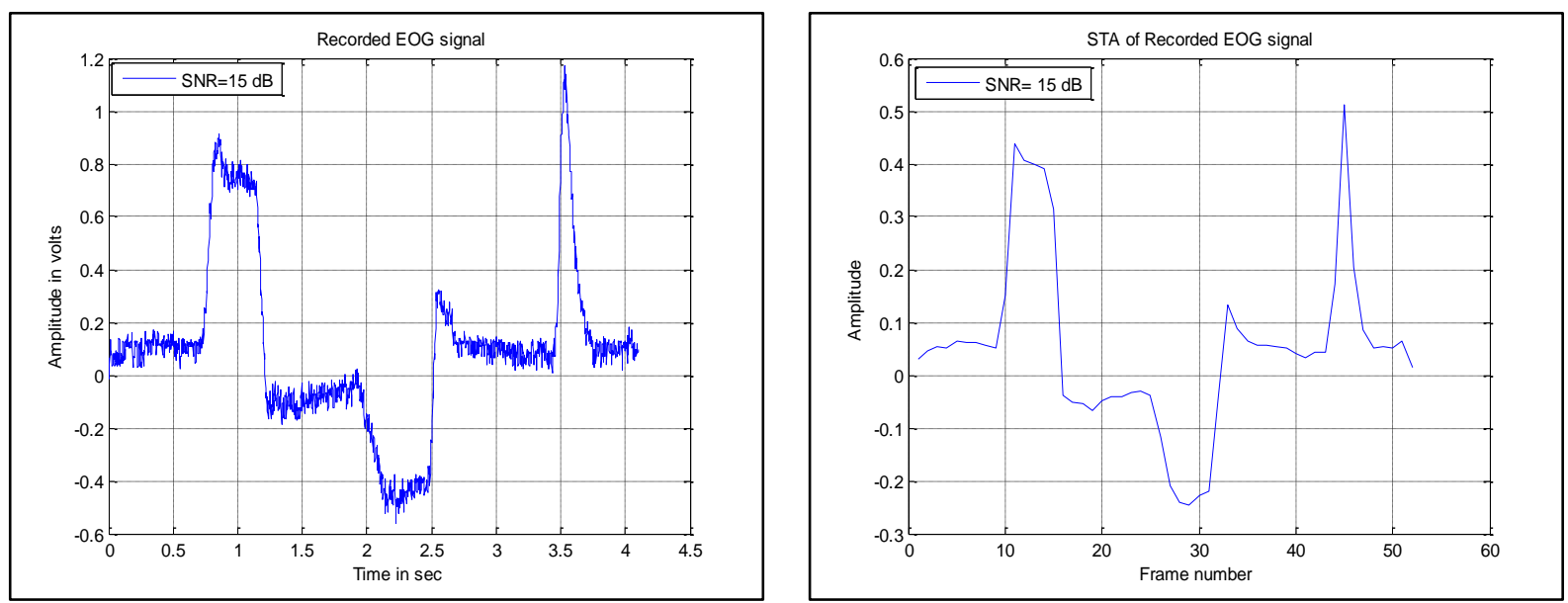

Fig. (9): Recorded EOG signal with $S N R=15 \mathrm{~dB}$ (Right), $S T A$ of recorded EOG signal with $S N R=15 \mathrm{~dB}(\mathrm{Left})$
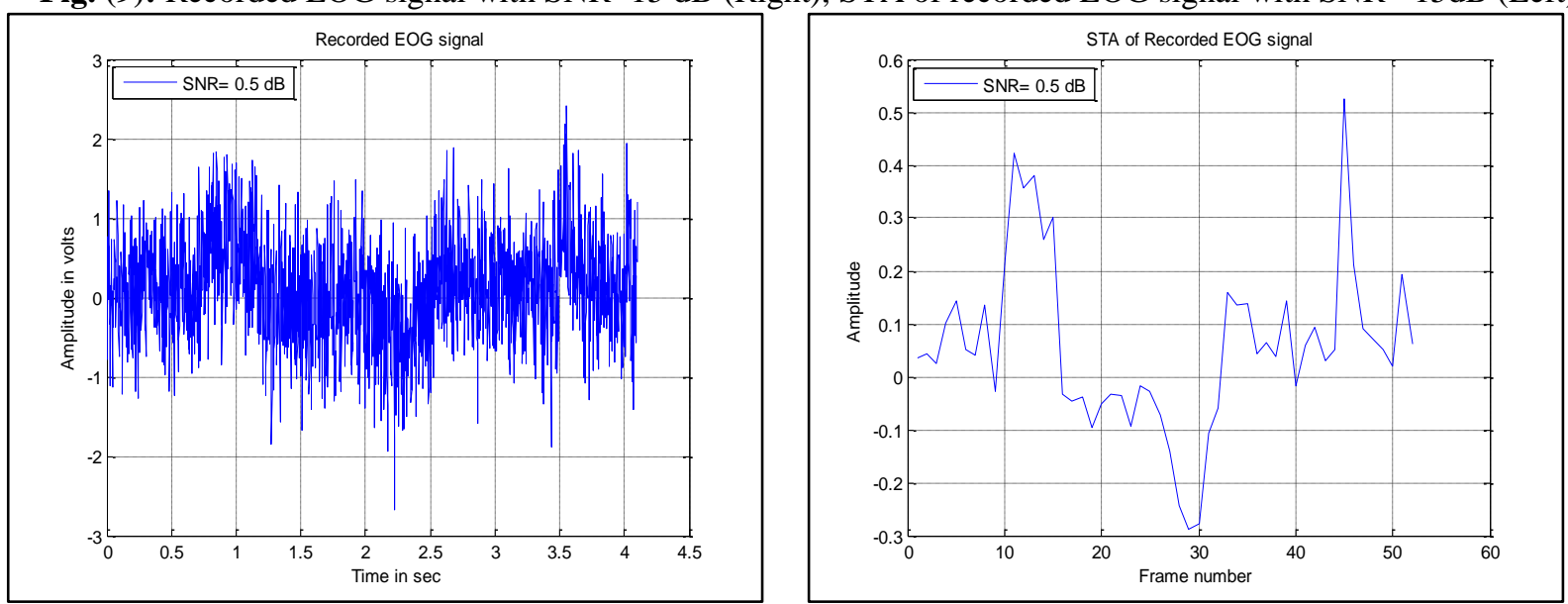

Fig. (10): Recorded EOG signal with $S N R=0.5 \mathrm{~dB}$ (Right), STA of recorded EOG signal with $\mathrm{SNR}=0.5 \mathrm{~dB}$ (Left)

As clearly shown in figure $9 \& 10$, the suggested STA method has reduced the impact of the noise significantly because this method is basically working as Moving Average filter per frame. In addition, this method is proposed to extract features from the EOG signals, these features are considered in the classification process as mentioned in subsection 5.2.

The performance of the algorithm was measured in terms of the Hit Rate percentage (HR\%) and False Rate percentage (FR\%). A good detection algorithm should have high HR\% and low FR\% .

$$
\begin{aligned}
H R \% & =\frac{\text { no. of correct detected pulses }}{\text { no. of actual pulses }} \times 100 \\
F R \% & =\frac{\text { no. of false detected pulses }}{\text { no. of actual pulses }} \times 100
\end{aligned}
$$

Thirty pulses of each eye movements are generated from the recorded EOG signals. These long sequences of pulses with different SNRs were applied to study the robustness of the suggested algorithm in noisy background. The performance of the algorithm in different SNRs of eye movement is located in Table 4. 
Table (4): Statistics of HIT rate and False rate

\begin{tabular}{|c|c|c|c|c|c|c|c|c|c|c|c|c|c|}
\hline \multirow{9}{*}{ 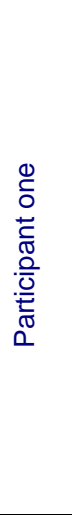 } & & \multicolumn{2}{|c|}{$S N R=15 d B$} & \multicolumn{2}{|c|}{$S N R=10 \mathrm{~dB}$} & \multicolumn{2}{|c|}{$\mathrm{SNR}=5 \mathrm{~dB}$} & \multicolumn{2}{|c|}{$S N R=2 d B$} & \multicolumn{2}{|c|}{$S N R=1 \mathrm{~dB}$} & \multicolumn{2}{|c|}{$\mathrm{SNR}=0.5 \mathrm{~dB}$} \\
\hline & movement & HR \% & $\begin{array}{l}\text { FR } \\
\%\end{array}$ & $\mathrm{HR} \%$ & $\begin{array}{l}\text { FR } \\
\%\end{array}$ & $\mathrm{HR} \%$ & $\begin{array}{l}\text { FR } \\
\%\end{array}$ & HR \% & $\mathrm{FR} \%$ & $\mathrm{HR} \%$ & $\mathrm{FR} \%$ & $\mathrm{HR} \%$ & $\mathrm{FR} \%$ \\
\hline & Blinking & $100 \%$ & $0 \%$ & $100 \%$ & $0 \%$ & $100 \%$ & $0 \%$ & $100 \%$ & $0 \%$ & 96.66 & $0 \%$ & 93.33 & $3.33 \%$ \\
\hline & & $100 \%$ & $0 \%$ & $100 \%$ & $0 \%$ & $100 \%$ & $0 \%$ & 9666 & $333 \%$ & $90 \%$ & $666 \%$ & 8666 & $10 \%$ \\
\hline & Uprocita & 10070 & 070 & (10 & 070 & (10 & 470 & $\%$ & 0.0070 & & 0.0070 & $\%$ & 1070 \\
\hline & Downward & $100 \%$ & $0 \%$ & $100 \%$ & $0 \%$ & $100 \%$ & $0 \%$ & 96.66 & $3.33 \%$ & 93.33 & $6.66 \%$ & 83.33 & $6.66 \%$ \\
\hline & & & & & & & & $\%$ & & $\%$ & & $\%$ & \\
\hline & Right & $100 \%$ & $0 \%$ & $100 \%$ & $0 \%$ & $100 \%$ & $0 \%$ & $100 \%$ & $0 \%$ & $100 \%$ & $0 \%$ & $\begin{array}{c}93.33 \\
\%\end{array}$ & $0 \%$ \\
\hline & Left & $100 \%$ & $0 \%$ & $100 \%$ & $0 \%$ & $100 \%$ & $0 \%$ & $100 \%$ & $0 \%$ & $100 \%$ & $0 \%$ & $90 \%$ & $0 \%$ \\
\hline & Eye & SNR $=$ & $\mathrm{dB}$ & SNR $=$ & $d B$ & SNR= & $d B$ & SNF & $\mathrm{dB}$ & SNR & $1 \mathrm{~dB}$ & SNR= & $5 \mathrm{~dB}$ \\
\hline & $\begin{array}{c}\text { movemen } \\
t\end{array}$ & $\mathrm{HR} \%$ & $\begin{array}{l}\text { FR } \\
\%\end{array}$ & $\mathrm{HR} \%$ & $\begin{array}{l}F R \\
\%\end{array}$ & $\mathrm{HR} \%$ & $\begin{array}{l}\text { FR } \\
\%\end{array}$ & HR \% & FR \% & $\mathrm{HR} \%$ & FR \% & $\mathrm{HR} \%$ & FR \% \\
\hline & Blinking & $100 \%$ & $0 \%$ & $100 \%$ & $0 \%$ & $100 \%$ & $0 \%$ & 96.66 & $3.33 \%$ & 96.66 & $6.66 \%$ & 96.66 & $6.66 \%$ \\
\hline & & & & & & & & $\%$ & & $\%$ & & $\%$ & \\
\hline ฮ్ & Upward & $100 \%$ & $0 \%$ & $100 \%$ & $0 \%$ & $100 \%$ & $0 \%$ & $100 \%$ & $0 \%$ & $100 \%$ & $3.33 \%$ & 93.33 & $6.66 \%$ \\
\hline$\overbrace{\frac{\pi}{\sigma}}$ & & & & & & 1000 & & 1000 & & & & & \\
\hline & Downwara & $100 \%$ & $0 \%$ & $100 \%$ & $0 \%$ & $100 \%$ & $0 \%$ & $100 \%$ & $0 \%$ & $\begin{array}{c}96.66 \\
\%\end{array}$ & $3.33 \%$ & $\begin{array}{c}93.33 \\
\%\end{array}$ & $6.66 \%$ \\
\hline & Right & $100 \%$ & $0 \%$ & $100 \%$ & $0 \%$ & $100 \%$ & $0 \%$ & 93.33 & $0 \%$ & $90 \%$ & $0 \%$ & 86.66 & $0 \%$ \\
\hline & & & & & & & & $\%$ & & & & $\%$ & \\
\hline & Left & $100 \%$ & $0 \%$ & $100 \%$ & $0 \%$ & $100 \%$ & $0 \%$ & 96.66 & $0 \%$ & 93.33 & $0 \%$ & $90 \%$ & $0 \%$ \\
\hline & & & & & & & & $\%$ & & $\%$ & & & \\
\hline & Eye & SNR= & & SNR $=$ & $\mathrm{dB}$ & SNR= & & SNF & dB & SNR & $1 \mathrm{~dB}$ & SNR= & $5 \mathrm{~dB}$ \\
\hline & $\begin{array}{c}\text { movemen } \\
t\end{array}$ & HR \% & $\begin{array}{l}\mathrm{FR} \\
\%\end{array}$ & $\mathrm{HR} \%$ & $\begin{array}{l}F R \\
\%\end{array}$ & $\mathrm{HR} \%$ & $\begin{array}{l}\text { FR } \\
\%\end{array}$ & HR \% & FR \% & $\mathrm{HR} \%$ & FR \% & $\mathrm{HR} \%$ & FR \% \\
\hline & Blinking & $100 \%$ & $0 \%$ & $100 \%$ & $0 \%$ & $100 \%$ & $0 \%$ & 96.66 & $3.33 \%$ & 93.33 & $3.33 \%$ & $80 \%$ & $10 \%$ \\
\hline$\underset{\Perp}{\Perp}$ & & & & & & & & $\%$ & & $\%$ & & & \\
\hline \pm & Upward & $100 \%$ & $0 \%$ & $100 \%$ & $0 \%$ & $100 \%$ & $0 \%$ & $100 \%$ & $0 \%$ & $100 \%$ & $0 \%$ & 96.66 & $3.33 \%$ \\
\hline 뜬 & & & & & & & & & & & & $\%$ & \\
\hline$\stackrel{0}{=}$ & Downward & $100 \%$ & $0 \%$ & $100 \%$ & $0 \%$ & $100 \%$ & $0 \%$ & 93.33 & $0 \%$ & $90 \%$ & $3.33 \%$ & 76.66 & $6.66 \%$ \\
\hline $\bar{\pi}$ & & & & & & & & $\%$ & & & & $\%$ & \\
\hline & Right & $100 \%$ & $0 \%$ & $100 \%$ & $0 \%$ & $100 \%$ & $0 \%$ & 96.66 & $0 \%$ & 96.66 & $0 \%$ & 86.66 & $0 \%$ \\
\hline & & & & & & & & $\%$ & & $\%$ & & $\%$ & \\
\hline & Left & $100 \%$ & $0 \%$ & $100 \%$ & $0 \%$ & $100 \%$ & $0 \%$ & $100 \%$ & $0 \%$ & $100 \%$ & $0 \%$ & 93.33 & $0 \%$ \\
\hline & & & & & & & & & & & & $\%$ & \\
\hline & & SNR= & dB & SNR= & $\mathrm{dB}$ & SNR= & & SNF & $\mathrm{dB}$ & SNR & $1 \mathrm{~dB}$ & SNR= & $5 \mathrm{~dB}$ \\
\hline & movemen & $\mathrm{HR} \%$ & FR & $\mathrm{HR} \%$ & FR & $\mathrm{HR} \%$ & FR & $\mathrm{HR} \%$ & FR \% & $\mathrm{HR} \%$ & FR \% & $\mathrm{HR} \%$ & FR \% \\
\hline & $t$ & & $\%$ & & $\%$ & & $\%$ & & & & & & \\
\hline & Blinking & $100 \%$ & $0 \%$ & $100 \%$ & $0 \%$ & $100 \%$ & $0 \%$ & $100 \%$ & $0 \%$ & 96.66 & $3.33 \%$ & 93.33 & $6.66 \%$ \\
\hline 亏ั้ & & & & & & & & & & $\%$ & & $\%$ & \\
\hline \pm & Upward & $100 \%$ & $0 \%$ & $100 \%$ & $0 \%$ & $100 \%$ & $0 \%$ & $100 \%$ & $3.33 \%$ & $100 \%$ & $3.33 \%$ & 96.66 & $6.66 \%$ \\
\hline$\frac{\pi}{0}$ & & & & & & & & & & & & $\%$ & \\
\hline$\stackrel{n}{ \pm}$ & Downward & $100 \%$ & $0 \%$ & $100 \%$ & $0 \%$ & $100 \%$ & $0 \%$ & 96.66 & $0 \%$ & 93.33 & $3.33 \%$ & 86.66 & $6.66 \%$ \\
\hline$\stackrel{\pi}{0}$ & & & & & & & & $\%$ & & $\%$ & & $\%$ & \\
\hline & Right & $100 \%$ & $0 \%$ & $100 \%$ & $0 \%$ & $100 \%$ & $0 \%$ & $100 \%$ & $0 \%$ & 96.66 & $3.33 \%$ & $90 \%$ & $10 \%$ \\
\hline & & & & & & & & & & $\%$ & & & \\
\hline & Left & $100 \%$ & $0 \%$ & $100 \%$ & $0 \%$ & $100 \%$ & $0 \%$ & $100 \%$ & $0 \%$ & 96.66 & $6.66 \%$ & 93.33 & $10 \%$ \\
\hline & & & & & & & & & & $\%$ & & $\%$ & \\
\hline 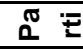 & Eye & SNR $=$ & & SNR $=$ & dB & SNR= & dB & SNF & $\mathrm{dB}$ & SNR & $1 \mathrm{~dB}$ & SNR= & $.5 \mathrm{~dB}$ \\
\hline
\end{tabular}




\begin{tabular}{|c|c|c|c|c|c|c|c|c|c|c|c|c|c|}
\hline & $\begin{array}{c}\text { movemen } \\
\mathbf{t}\end{array}$ & HR \% & $\begin{array}{l}\text { FR } \\
\% \\
\end{array}$ & $\mathrm{HR} \%$ & $\begin{array}{l}\text { FR } \\
\% \\
\end{array}$ & $\mathrm{HR} \%$ & $\begin{array}{l}\text { FR } \\
\% \\
\end{array}$ & HR \% & FR \% & $\mathrm{HR} \%$ & FR \% & $\mathrm{HR} \%$ & FR \% \\
\hline & Blinking & $100 \%$ & $0 \%$ & $100 \%$ & $0 \%$ & $100 \%$ & $0 \%$ & $100 \%$ & $0 \%$ & $\begin{array}{c}96.66 \\
\% \\
\end{array}$ & $0 \%$ & $\begin{array}{c}93.33 \\
\% \\
\end{array}$ & $3.33 \%$ \\
\hline & Upward & $100 \%$ & $0 \%$ & $100 \%$ & $0 \%$ & $100 \%$ & $0 \%$ & $\begin{array}{c}96.66 \\
\% \\
\end{array}$ & $3.33 \%$ & $90 \%$ & $6.66 \%$ & $\begin{array}{c}86.66 \\
\% \\
\end{array}$ & $10 \%$ \\
\hline & Downward & $100 \%$ & $0 \%$ & $100 \%$ & $0 \%$ & $100 \%$ & $0 \%$ & $100 \%$ & $0 \%$ & $\begin{array}{c}96.66 \\
\% \\
\end{array}$ & $3.33 \%$ & $\begin{array}{c}93.33 \\
\% \\
\end{array}$ & $6.66 \%$ \\
\hline & Right & $100 \%$ & $0 \%$ & $100 \%$ & $0 \%$ & $100 \%$ & $0 \%$ & $\begin{array}{c}93.33 \\
\% \\
\end{array}$ & $0 \%$ & $90 \%$ & $0 \%$ & $\begin{array}{c}86.66 \\
\% \\
\end{array}$ & $0 \%$ \\
\hline & Left & $100 \%$ & $0 \%$ & $100 \%$ & $0 \%$ & $100 \%$ & $0 \%$ & $100 \%$ & $0 \%$ & $100 \%$ & $0 \%$ & $\begin{array}{c}93.33 \\
\% \\
\end{array}$ & $0 \%$ \\
\hline \multirow{7}{*}{ 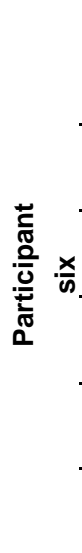 } & & \multicolumn{2}{|c|}{ SNR=15dB } & \multicolumn{2}{|c|}{ SNR=10 dB } & \multicolumn{2}{|c|}{ SNR $=5 \mathrm{~dB}$} & \multicolumn{2}{|c|}{ SNR=2 dB } & \multicolumn{2}{|c|}{ SNR $=1 \mathrm{~dB}$} & \multicolumn{2}{|c|}{ SNR $=0.5 \mathrm{~dB}$} \\
\hline & $\begin{array}{c}\text { movemen } \\
\mathbf{t}\end{array}$ & HR \% & $\begin{array}{l}\text { FR } \\
\% \\
\end{array}$ & $\mathrm{HR} \%$ & $\begin{array}{l}\text { FR } \\
\% \\
\end{array}$ & $\mathrm{HR} \%$ & $\begin{array}{l}\text { FR } \\
\% \\
\end{array}$ & $\mathrm{HR} \%$ & FR \% & $\mathrm{HR} \%$ & FR \% & $\mathrm{HR} \%$ & FR \% \\
\hline & Blinking & $100 \%$ & $0 \%$ & $100 \%$ & $0 \%$ & $100 \%$ & $0 \%$ & $\begin{array}{c}96.66 \\
\% \\
\end{array}$ & $3.33 \%$ & $\begin{array}{c}96.66 \\
\% \\
\end{array}$ & $6.66 \%$ & $\begin{array}{c}96.66 \\
\% \\
\end{array}$ & $6.66 \%$ \\
\hline & Upward & $100 \%$ & $0 \%$ & $100 \%$ & $0 \%$ & $100 \%$ & $0 \%$ & $100 \%$ & $0 \%$ & $100 \%$ & $0 \%$ & $\begin{array}{c}96.66 \\
\% \\
\end{array}$ & $3.33 \%$ \\
\hline & Downward & $100 \%$ & $0 \%$ & $100 \%$ & $0 \%$ & $100 \%$ & $0 \%$ & $\begin{array}{c}93.33 \\
\% \\
\end{array}$ & $0 \%$ & $90 \%$ & $3.33 \%$ & $\begin{array}{c}76.66 \\
\% \\
\end{array}$ & $6.66 \%$ \\
\hline & Right & $100 \%$ & $0 \%$ & $100 \%$ & $0 \%$ & $100 \%$ & $0 \%$ & $100 \%$ & $0 \%$ & $\begin{array}{c}96.66 \\
\% \\
\end{array}$ & $3.33 \%$ & $90 \%$ & $10 \%$ \\
\hline & Left & $100 \%$ & $0 \%$ & $100 \%$ & $0 \%$ & $100 \%$ & $0 \%$ & $100 \%$ & $0 \%$ & $100 \%$ & $0 \%$ & $\begin{array}{c}93.33 \\
\%\end{array}$ & $0 \%$ \\
\hline
\end{tabular}

From Table 4, the results show that the suggested algorithm has an excellent performance ( $\mathrm{HR}=100 \%$ and $\mathrm{FR}=0 \%$, when the $\mathrm{SNR}$ is between $15 \mathrm{~dB}$ and $5 \mathrm{~dB}$. Furthermore, in the case of $\mathrm{SNR}=2 \mathrm{~dB}$, the performance of the algorithm is still acceptable with minimum $\mathrm{HR}=93.33 \%$ and maximum $\mathrm{FR}=3.33 \%$. The average performances in case of $\mathrm{SNR}=2 \mathrm{~dB}, 1 \mathrm{~dB}$ and $0.5 \mathrm{~dB}$ were calculated by averaging the Hit rate of six participants. In general, the average performance of STA method in case of SNR=2 dB for blinking ,looking up, down and left is better comparing with mathematical morphology method as shown in table

Table (5): Comparison between STA method and mathematical morphology method

\begin{tabular}{lcccc}
\hline Eye movements & $\begin{array}{c}\text { HR\% of STA method } \\
(\mathrm{SNR}=2 \mathrm{~dB})\end{array}$ & $\begin{array}{c}\text { HR\% of STA method } \\
(\mathrm{SNR}=1 \mathrm{~dB})\end{array}$ & $\begin{array}{c}\text { HR\% of STA method } \\
(\mathrm{SNR}=0.5 \mathrm{~dB})\end{array}$ & $\begin{array}{c}\text { HR\% of mathematical morphology } \\
\text { method (Jiang \& Zhou, 2013) }\end{array}$ \\
\hline Blinking & $98.33 \%$ & $96.10 \%$ & $92.22 \%$ & $86.50 \%$ \\
\hline Looking Up & $98.88 \%$ & $96.66 \%$ & $92.77 \%$ & $93.49 \%$ \\
\hline Looking Down & $96.66 \%$ & $93.33 \%$ & $84.99 \%$ & $91.94 \%$ \\
\hline Looking Right & $97.22 \%$ & $94.99 \%$ & $88.88 \%$ & $97.56 \%$ \\
\hline Looking Left & $99.44 \%$ & $98.33 \%$ & $92.22 \%$ & $97.83 \%$ \\
\hline
\end{tabular}

\section{CONCLUSION}

In this paper, the proposed algorithm succeeds in classifying five eye movements such as looking up, down, right, left and blinking. The main advantage of this algorithm is the short-time averaging per frame method which is used to extract information from EOG signals. In addition, it also acts as a moving average filter. This feature makes the algorithm robust in noisy environments without any De-nosing stage. In general, it found that the performance of this algorithm is considerably accepted and it gives efficient and consistent results with minimum noise level. Besides, Using simple methods for classification make this algorithm easy to be implemented for online applications such as human machine interface. 


\section{REFERENCES}

- Prasad, R. V., Sangwan, A., Jamadagni, H., M.C, C., \& Sah, R. (2002). Comparison of Voice Activity Detection Algorithms for VoIP. Proceedings of the Seventh International Symposium on Computers and Communications (pp. 1-6). Singapore: IEEE.

- Estrany, B., Fuster, P., Garcia, A., \& Luo, Y. (2008). Human computer interface by EOG tracking. Proceedings of the 1st ACM International Conference on Pervasive Technologies Related to Assistive Environment (pp. 16-23). Athens, Greece: PETRA.

- Estrany, B., Fuster, P., Garcia, A., \& Luo , Y. (2009). EOG signal processing and analysis for controlling computer by eye movements. Proceedings of the 2nd International Conference on Pervasive Technologies Related to Assistive Environments (pp. 9-13). Corfu, Greece: PETRA .

- Gold, B., \& Morgan, N. (1999). Speech and Audio Signal Processing: Processing and Perception of Speech and Music (2nd ed.). span: John Wiley Publications.

- Iáñez, E., Úbeda, A., \& Azorín, J. (2011). Multimodal human-machine interface based on a braincomputer interface and an electrooculography interface. Proceeding Engineering in Medicine and Biology Society (pp. 4572-4575). Annual International Conference of the IEEE.

- Jiang, P., \& Zhou, R. (2013). De-noising and recognition of EOG signal based on mathematical morphology. 2013 Sixth International Symposium on Computational Intelligence and Design, (pp. 351-354). Hangzhou, China.

- Jose, J. (2013). Development of EOG Based Human Machine Interface Control System for Motorized Wheelchair (Unpublised Master thesis). National Institute Of Technology Rourkela, Odisha, India
- Kim, M., \& Yoon, G. (2013). Control Signal from EOG Analysis and Its Application. International Journal of Electrical, Computer, Energetic, Electronic and Communication Engineering, 7(10), 1352-1355.

- Lv, Z., Wu, X.-p., Li, M., \& Zhang, D. (2010). A novel eye movement detection algorithm for EOG driven human computer interface. Pattern Recognition Letters, 1041-1047.

- Mala, S., \& Latha, K. (2016). ELECTROOCULOGRAPHY DE-NOISING: WAVELET BASED APPROACH TO REDUCE NOISE. Iternational Journal of Adavnced Egineering Technology, 6(2), 482-487.

- Malik, A., \& Ahmad , J. (2007). Retina Based Mouse Control (RBMC). World Academy of Science, Engineering and Technology, 1(7), 318-321.

- Merino, M., Rivera, O., Gómez, I., Molina, A., \& Dorronzoro, E. (2010). A Method of EOG Signal Processing to Detect the Direction of Eye Movements. 2010 First International Conference on Sensor Device Technologies and Applications. Venice, Italy.

- Pollak, P., Sovka, P., \& Jan, U. (1993). Noise suppression system for a car. Third European Conference on Speech Communication and Technology (pp. 1073-1076). Berlin, Germany: EUROSPEECH.

- Rajesh A, N., S., C., T., A., \& K., S. (2012). Denoising EOG Signal using Stationary Wavelet Transform. MEASUREMENT SCIENCE REVIEW, 12(2), 46-51.

- Sangwan, A., M.C, C., Jamadagni, H., Sah, R., Prasad, R., \& Gaurav, V. (2002). VAD Techniques for Real-Time Speech Transmission on the Internet. High Speed Networks and Multimedia Communications 5th IEEE International Conference (pp. 1-6). IEEE. 